\title{
Taxane Analogue TPI 287
}

National Cancer Institute

\section{Source}

National Cancer Institute. Taxane Analogue TPI 287. NCI Thesaurus. Code C48424.

A synthetic, third generation taxane with potential antineoplastic activity. TPI 287 binds to

tubulin and stabilizes microtubules, resulting in inhibition of microtubule

assembly/disassembly dynamics, cell cycle arrest at the G2/M phase, and apoptosis. 